\title{
Cosmic ray propagation after AMS-02
}

\author{
Qiang Yuan*1, Su-Jie Lin ${ }^{2}$, Kun Fang ${ }^{2}$, Xiao-Jun $\mathrm{Bi}^{2}$ \\ ${ }^{1}$ Key Laboratory of Dark Matter and Space Astronomy, Purple Mountain Observatory, Chinese \\ Academy of Sciences, Nanjing 210008, P.R.China \\ ${ }^{2}$ Key Laboratory of Particle Astrophysics, Institute of High Energy Physics, Chinese Academy of \\ Sciences, Beijing 100049, P.R.China \\ E-mail: yuangepmo.ac.cn
}

The precise measurements of cosmic ray (CR) fluxes and ratios by AMS-02 enable significantly improved constraints on the propagation models of cosmic rays. Recently the AMS-02 collaboration reported high precision measurement of the Boron-to-Carbon ratio $(\mathrm{B} / \mathrm{C})$. Together with the proton fluxes and their time evolutions by AMS-02 and PAMELA at earlier time, we re-visit the propagation of CRs in the Milky Way. These sets of data enable us to constrain the parameters of both the injection and propagation simultaneously, as well as a better characterization of the solar modulation effect with the time-evolved spectra. We study a comprehensive set of propagation models, with/without the reacceleration or convection effect. We find that generally reacceleration models fit the proton and $\mathrm{B} / \mathrm{C}$ data better than non-reacceleration ones. However, the reacceleration models over-predict low energy secondary positrons when compared with the data. The rigidity dependence slope of the diffusion coefficient, $\delta$, is found to be about $0.38-0.50$ for reacceleration models, which slightly favors the Kraichnan type of interstellar turbulence but can not exclude the Kolmogorov case turbulence. The plain diffusion and diffusion-convection models are consistent with the positron data, but fit the nuclei data poorly. Our results suggest that the propagation of nuclei, in either the Milky Way or the solar heliosphere, may be different from that of leptons.

35th International Cosmic Ray Conference - ICRC2017

10-20 July, 2017

Bexco, Busan, Korea

\footnotetext{
* Speaker.
} 


\section{Introduction}

It has been well established that charged cosmic rays (CRs) propagate diffusively in the Milky Way. This is directly supported by the tiny anisotropies of arrival directions of CRs, as well as the abundance of radioactive isotopes. There are generally two types of nuclei in CRs: the primary class such as $p, \mathrm{He}, \mathrm{C}, \mathrm{O}, \mathrm{Fe}$ which are abundant products of stellar evolution, and the secondary class such as $\mathrm{Li}, \mathrm{Be}, \mathrm{B}, \mathrm{Sc}, \mathrm{Ti}, \mathrm{V}, \mathrm{Cr}, \mathrm{Mn}$ which are rare in stellar nucleosynthesis. These secondary nuclei in CRs are thus dominately from the fragmentation of primary CRs due to their collision with the interstellar gas in the Milky Way. The ratios between secondary nuclei and their parent nuclei, for example $\mathrm{B} / \mathrm{C}$ and $(\mathrm{Sc}+\mathrm{Ti}+\mathrm{V}+\mathrm{Cr}+\mathrm{Mn}) / \mathrm{Fe}$, probe the journey of primary particles during their propagation ${ }^{1}$. In addition, unstable isotopes such as ${ }^{10} \mathrm{Be}$ (half-life $\tau=1.39 \times 10^{6} \mathrm{yr}$ ) and ${ }^{26} \mathrm{Al}$ ( $\tau=7.17 \times 10^{5} \mathrm{yr}$ ) measure the residual time of CRs in the Milky Way halo. Therefore, data of the secondary-to-primary ratios and unstable-to-stable ratios are widely used to probe the propagation of CRs $[1,2]$.

Although quite a lot of efforts had been made to measure the $\mathrm{B} / \mathrm{C}$ ratio (e.g., $[3,4,5])$, the uncertainties are large which render good constraints on the propagation parameters [6, 7, 8, 9]. Very recently, the Alpha Magnetic Spectrometer 02 (AMS-02) onboard the International Space Station reported the very precise measurements of the $\mathrm{CR} B / \mathrm{C}$ ratio up to rigidities of $2.6 \mathrm{TV}$ [10]. The AMS-02 data show that the $\mathrm{B} / \mathrm{C}$ ratio follows a single power-law form of $R^{-1 / 3}$ above $\sim 60 \mathrm{GV}$, without any significant structures. The energy (or rigidity) dependence of the B/C ratio suggests that CRs propagate diffusively in the interstellar turbulence with the Kolmogorov type power spectrum.

However, the rigidity dependence of the $\mathrm{B} / \mathrm{C}$ data may not be solely determined by the diffusion process. The possible convective transportation and/or reacceleration of particles modifies the rigidity dependence of the $\mathrm{B} / \mathrm{C}$ ratio, especially at low energies. This in turn affects the determination of the rigidity dependence of the diffusion coefficient. Therefore a detailed analysis of various propagation models based on the new data is desirable. Another possible issue when studying the propagation parameters is the entanglement with the solar modulation effect. The time-evolved spectra of protons by PAMELA [11] and AMS-02 [12] are helpful in breaking the entanglement, at least partly. Here we report the study of the CR propagation models and constrain the propagation parameters using the latest $\mathrm{B} / \mathrm{C}$ data by AMS-02 and time-evolved proton spectra by PAMELA and AMS-02, based on a global fitting method [13]. The ACE observations of the B/C during the same period of AMS-02 data are also included [13], which are helpful in regulating the low energy behavior of the $\mathrm{B} / \mathrm{C}$.

\section{CosRayMC - a Markov Chain Monte Carlo tool for CR propagation}

The propagation of CRs in the Milky Way is a complicated, multi-parameter problem. Some of the model parameters are degenerate (e.g., the diffusion coefficient and the height of the propagation halo), and can not be well constrained [6]. Therefore, an efficient way to survey the high-

\footnotetext{
${ }^{1}$ Spallation may also occur at the source during the acceleration process, and the secondary particles may get accelerated at the same time. However, diffuse $\gamma$-ray observations suggest that most of such interactions should take place during the propagation instead of surrounding the source.
} 
dimensional parameter space is very useful. The Markov Chain Monte Carlo (MCMC) method is widely applied in cosmology and astrophysics in model fittings and parameter inference $[14,15]$.

We have developed a tool, CosRayMC, through integrating the numerical propagation code GALPROP $[16,17]$ with the MCMC sampler $[18,19]$. The Markov chain is generated following the Metropolis-Hastings algorithm, which is a propose-and-accept process. The acceptance probability of a proposed step in the parameter space depends on the probability ratio between this proposed new point and the former one. The stationary distribution of the sample of the chain approaches the target probability distribution.

\section{Model configuration and paramaters}

The geometry of the propagation volume is usually characterized by a cylinder with half height of $z_{h}$. The diffusion coefficient depends on particle rigidity and is usually parameterized as a power-law form $D_{x x}=D_{0} \beta\left(R / R_{0}\right)^{\delta}$, where $\beta$ is the velocity in unit of light speed. The power-law index $\delta$ depends on the property of the interstellar turbulence, which is left to be a free parameter in the fitting. Sometimes a modification of the velocity dependence of the diffusion coefficient, with $\beta \rightarrow \beta^{\eta}$, was introduced to account for potential resonant interaction of CR particles and the magnetohydrodynamic (MHD) waves which results in dissipation of such waves [20].

Particles may experience convective transportation and be reaccelerated due to scattering with random MHD waves [2]. The convection is assumed to linearly increase with distance away from the disk, i.e., $\mathbf{V}_{\mathbf{c}}=\mathbf{V}_{\mathbf{0}}+\mathbf{z} \cdot \mathrm{d} V_{c} / \mathrm{d} z$. To maintain the continuous condition we adopt $\mathbf{V}_{\mathbf{0}}=0$. The stochastic reacceleration is characterized by a diffusion in the momentum space, with a momentum diffusion coefficient $D_{p p}$ related to $D_{x x}$ via the Alfven velocity of the magnetized medium. The spatial distribution of CR sources is assumed to follow the distribution of supernova remnants. The injection spectrum of CR nuclei is assumed to be a broken power-law function of rigidity, with spectral indices $v_{1}\left(v_{2}\right)$ below (above) break rigidity $R_{\mathrm{br}}$. We further need to introduce a normalization parameter $A_{p}$ to match the local proton fluxes.

The solar modulation affects the low energy spectrum significantly. We adopt the force field approximation of the solar modulation effect [21]. However, since we use data at different time which correspond to different modulation magnitude, we employ a linear evolution of the modulation potential with respect to the number of sunspots, $\Phi=\Phi_{0}+\Phi_{1} \cdot N(t) / N_{\max }$, to describe the time-dependence of solar modulation.

Several model configurations are discussed, including 1) the plain diffusion (PD) model, 2) the diffusion convection (DC) model, 3) the diffusion convection model with a break of the power-law form of the diffusion coefficient (DC2), 4) the diffusion reacceleration model (DR), 5) the diffusion reacceleration with $\beta^{\eta}$ term (DR2), and 6) the diffusion reacceleration convection (DRC) model. The full parameter space is thus $\left(D_{0}, \delta, z_{h}, v_{A}, \mathrm{~d} V_{c} / \mathrm{d} z, R_{0}, \eta, A_{p}, v_{1}, v_{2}, R_{\mathrm{br}}, \Phi_{0}, \Phi_{1}\right)$.

\section{Results}

The fitting results of all the six models are summarized in Table 1. From the fittings we find that the models without reacceleration generally give poorer fittings to the data than the recceleration models. The $\chi^{2}$ values of non-reacceleration models suggest fitting $p$-values of $7.8 \times 10^{-16}, 4.3 \times$ 
Table 1: Posterior mean and 68\% credible uncertainties of the model parameters

\begin{tabular}{|c|c|c|c|c|c|c|}
\hline Parameter & PD & $\mathrm{DC}$ & DC2 & DR & DR2 & DRC \\
\hline$D_{0}\left(10^{28} \mathrm{~cm}^{2} \mathrm{~s}^{-1}\right)$ & $5.29 \pm 0.51$ & $4.20 \pm 0.30$ & $4.95 \pm 0.35$ & $7.24 \pm 0.97$ & $4.16 \pm 0.57$ & $6.14 \pm 0.45$ \\
\hline$\delta$ & $0.471 \pm 0.006$ & $0.588 \pm 0.013$ & $0.591 \pm 0.011$ & $0.380 \pm 0.007$ & $0.500 \pm 0.012$ & $0.478 \pm 0.013$ \\
\hline$z_{h}(\mathrm{kpc})$ & $6.61 \pm 0.98$ & $10.90 \pm 1.60$ & $10.80 \pm 1.30$ & $5.93 \pm 1.13$ & $5.02 \pm 0.86$ & $12.70 \pm 1.40$ \\
\hline$v_{A}\left(\mathrm{~km} \mathrm{~s}^{-1}\right)$ & - & - & - & $38.5 \pm 1.3$ & $18.4 \pm 2.0$ & $43.2 \pm 1.2$ \\
\hline$d V_{c} / d z\left(\mathrm{~km} \mathrm{~s}^{-1} \mathrm{kpc}^{-1}\right)$ & - & $5.36 \pm 0.64$ & $5.02 \pm 0.55$ & - & - & $11.99 \pm 1.26$ \\
\hline$R_{0}(\mathrm{GV})$ & - & - & $5.29 \pm 0.23$ & - & - & - \\
\hline$\eta$ & - & - & - & - & $-1.28 \pm 0.22$ & - \\
\hline $\log \left(A_{p}\right)$ & $-8.334 \pm 0.003$ & $-8.334 \pm 0.003$ & $-8.336 \pm 0.003$ & $-8.347 \pm 0.002$ & $-8.334 \pm 0.002$ & $-8.345 \pm 0.002$ \\
\hline$v_{1}$ & $2.44 \pm 0.01$ & $2.45 \pm 0.01$ & $2.43 \pm 0.01$ & $1.69 \pm 0.02$ & $2.04 \pm 0.03$ & $1.82 \pm 0.02$ \\
\hline$v_{2}$ & $2.34 \pm 0.03$ & $2.30 \pm 0.01$ & $2.30 \pm 0.01$ & $2.37 \pm 0.01$ & $2.33 \pm 0.01$ & $2.37 \pm 0.01$ \\
\hline $\log \left(R_{b r} / \mathrm{MV}\right)$ & $5.06 \pm 0.13$ & $4.82 \pm 0.05$ & $4.78 \pm 0.06$ & $4.11 \pm 0.02$ & $4.03 \pm 0.03$ & $4.22 \pm 0.03$ \\
\hline$\Phi_{0}(\mathrm{GV})$ & $0.595 \pm 0.005$ & $0.537 \pm 0.006$ & $0.419 \pm 0.005$ & $0.180 \pm 0.008$ & $0.290 \pm 0.014$ & $0.220 \pm 0.008$ \\
\hline$\Phi_{1}(\mathrm{GV})$ & $0.495 \pm 0.011$ & $0.485 \pm 0.011$ & $0.472 \pm 0.012$ & $0.487 \pm 0.011$ & $0.485 \pm 0.011$ & $0.482 \pm 0.013$ \\
\hline$\chi^{2} /$ dof & $748.6 / 463$ & $591.0 / 462$ & $494.6 / 461$ & $438.8 / 462$ & $341.0 / 461$ & $380.5 / 461$ \\
\hline
\end{tabular}

$10^{-5}$, and 0.14 for the PD, DC, and DC2 models, respectively. This is mainly due to the fact that the reacceleration effect can better reproduce the peak of the $\mathrm{B} / \mathrm{C}$ ratio at $\sim \mathrm{GeV} / \mathrm{n}$ (see Figure 1).

The propagation parameters are relatively well constrained. Figure 2 shows an example of the 1-dimensional and 2-dimensional distributions of the key propagation parameters for the DR model. There is a clear degeneracy between $D_{0}$ and $\delta$, due probably to the relatively large uncertainties of the unstable-to-stable secondary ratio. Nevertheless, the constraints on the model parameters are improved compared with previous studies [7]. For instance, the posterior 95\% range of $z_{h}$ is found to be $[3.7,8.2] \mathrm{kpc}$, which is $[3.2,8.6] \mathrm{kpc}$ in Ref. [7]. A recent study using different data sets of AMS-02 found somehow different but still consistent values of $[6.2,9.4] \mathrm{kpc}$ [24]. The slope $\delta$ is about 0.38 for the DR model, and about 0.5 for the DR2/DRC models. It is currently difficult to distinguish the Kolmogorov model $(\delta=1 / 3)$ from the Kraichnan model $(\delta=1 / 2)$ of the interstellar turbulence.

The expected fluxes of secondary positrons are shown in Figure 3. Different from the fittings of nuclei, we find that the non-acceleration models predict positrons lower than the data, while reacceleration models over-produce positrons below $\sim 10 \mathrm{GeV}$. We note that the low energy results (for both the $\mathrm{B} / \mathrm{C}$ and positrons) are affected by the solar modulation effect. Therefore this result may indicate that the production and propagation of positrons may be significantly different from that of CR nuclei, either in the Milky Way or in the heliopshere. For all the models the high energy positron spectra are softer than the data, implying the existence of exotic primary positron sources.

Figure 4 shows the local interstellar (LIS) proton spectra, compared with the Voyager 1 measurements which is expected to be less affected by the solar modulation [23]. It is shown that the DR2 model may give roughly consistent LIS spectra with the data. The DR and DRC models under-predict the LIS proton fluxes, and all the non-reacceleration models over-predict the LIS proton fluxes. It would be necessary for future work to include the Voyager data in the fittings to finally compare different models.

\section{Conclusion}

Using the precise measurements of the $\mathrm{B} / \mathrm{C}$ ratio and time-dependent proton fluxes by AMS02 and PAMELA, we constrain the propagation models and parameters of CRs. A systematic 

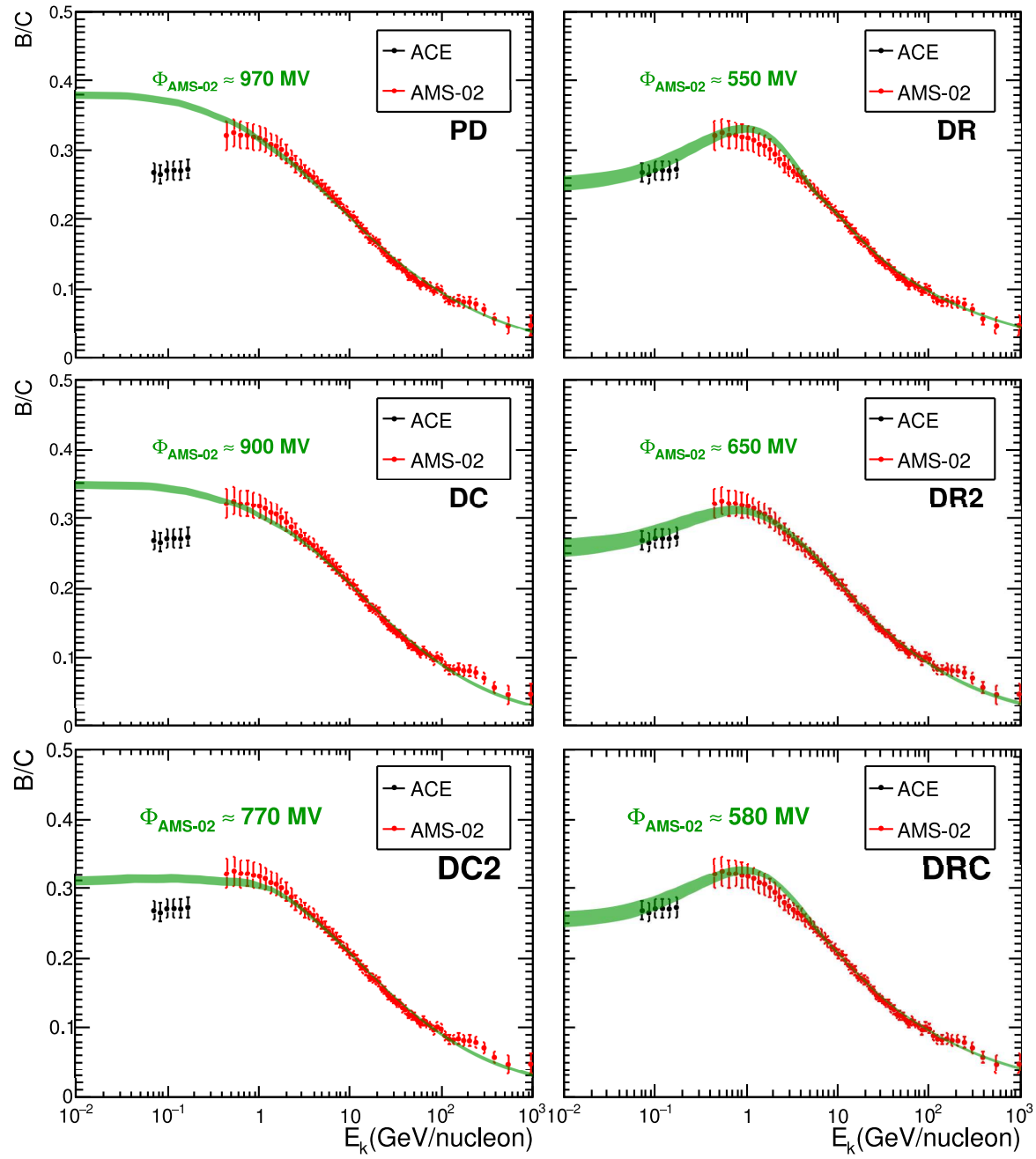

Figure 1: $2 \sigma$ bands of the $\mathrm{B} / \mathrm{C}$ ratios for different $\mathrm{PD}$ propagation models. The observational data are from: ACE [13] and AMS-02 [10].

investigation of different propagation settings with/without the convection and/or reacceleration effects are discussed. Our major conclusion is that the models with reacceleration effect are in good consistent with the CR nuclei data but predict too many low energy positrons, while the nonacceleration models fit the CR nuclei data poorly but are conpatible with the positron data. Our results imply that the propagation may be different between nuclei and leptons.

\section{References}

[1] T. K. Gaisser, Cosmic rays and particle physics, Cambridge University Press, Cambridge 1990.

[2] A. W. Strong, I. V. Moskalenko and V. S. Ptuskin, Cosmic-ray propagation and interactions in the Galaxy, Ann. Rev. Nucl. Part. Sci. 57 (2007) 285 [astro-ph/0701517].

[3] J. J. Engelmann, P. Ferrando, A. Soutoul, P. Goret and E. Juliusson, Charge composition and energy spectra of cosmic-ray for elements from Be to NI - Results from HEAO-3-C2, Astron. Astrophys. 233 (1990) 96. 


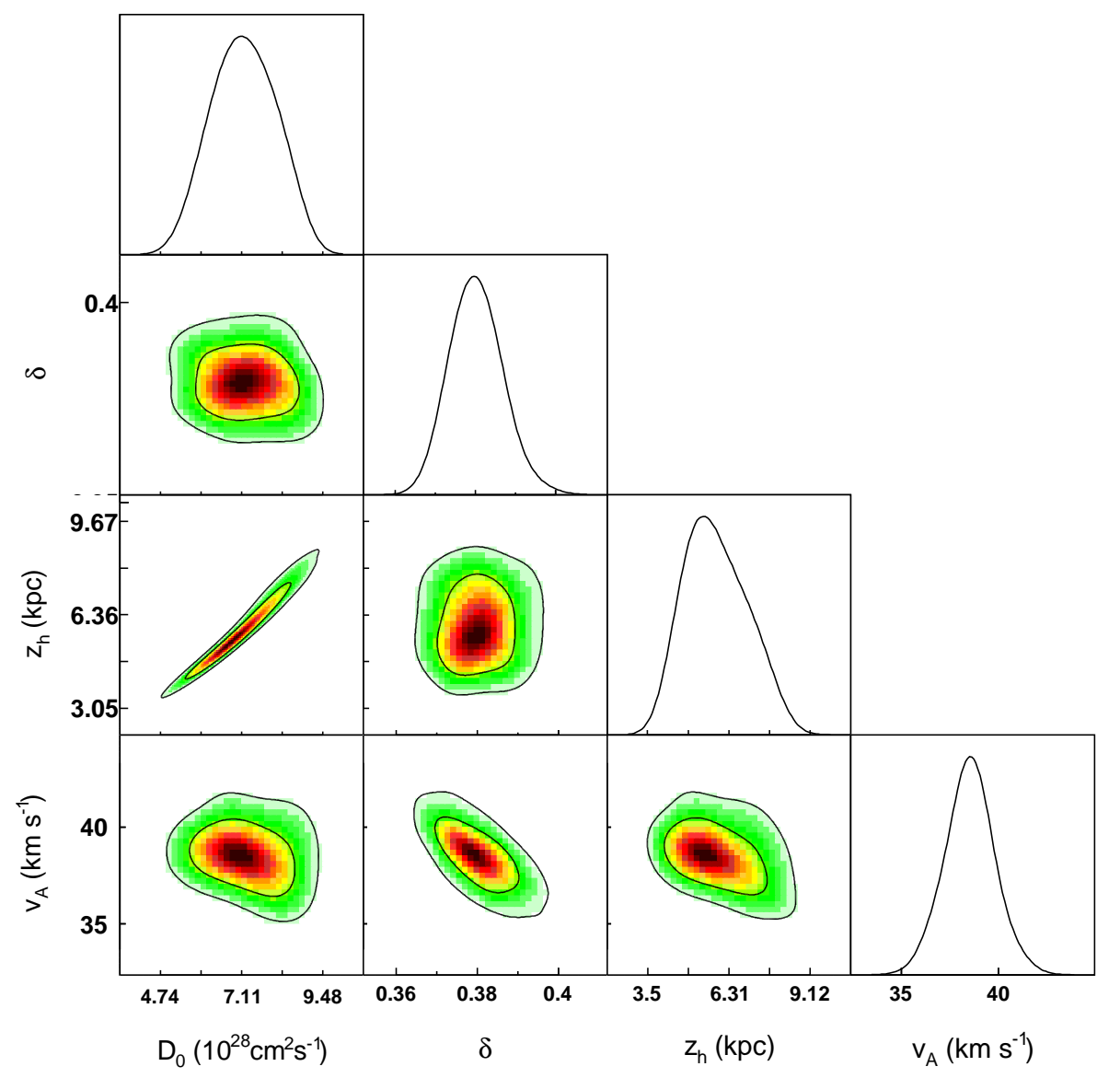

Figure 2: Triangle plot of the distributions of the parameters for the DR model. Only the four key propagation parameters are shown $\left(D_{0}, \delta, z_{h}, v_{A}\right)$.

[4] J. S. George et al., Elemental Composition and Energy Spectra of Galactic Cosmic Rays During Solar Cycle 23, Astrophys. J. 698 (2009) 1666.

[5] O. Adriani et al., Measurement of boron and carbon fluxes in cosmic rays with the PAMELA experiment, Astrophys. J. 791 (2014) 93 [arXiv:1407.1657].

[6] D. Maurin, F. Donato, R. Taillet and P. Salati, Cosmic rays below z=30 in a diffusion model: new constraints on propagation parameters, Astrophys. J. 555 (2001) 585 [astro-ph/0101231].

[7] R. Trotta, G. Johannesson, I. V. Moskalenko, T. A. Porter, R. R. de Austri and A. W. Strong, Constraints on cosmic-ray propagation models from a global Bayesian analysis, Astrophys. J. $\mathbf{7 2 9}$ (2011) 106 [arXiv:1011.0037].

[8] H. B. Jin, Y. L. Wu and Y. F. Zhou, Cosmic ray propagation and dark matter in light of the latest AMS-02 data, J. Cosmol. Astropart. Phys. 09 (2015) 049 [arXiv:1410.0171].

[9] J. Feng, N. Tomassetti and A. Oliva, Bayesian analysis of spatial-dependent cosmic-ray propagation: astrophysical background of antiprotons and positrons, Phys. Rev. D 94 (2016) 123007

[arXiv:1610.06182]. 

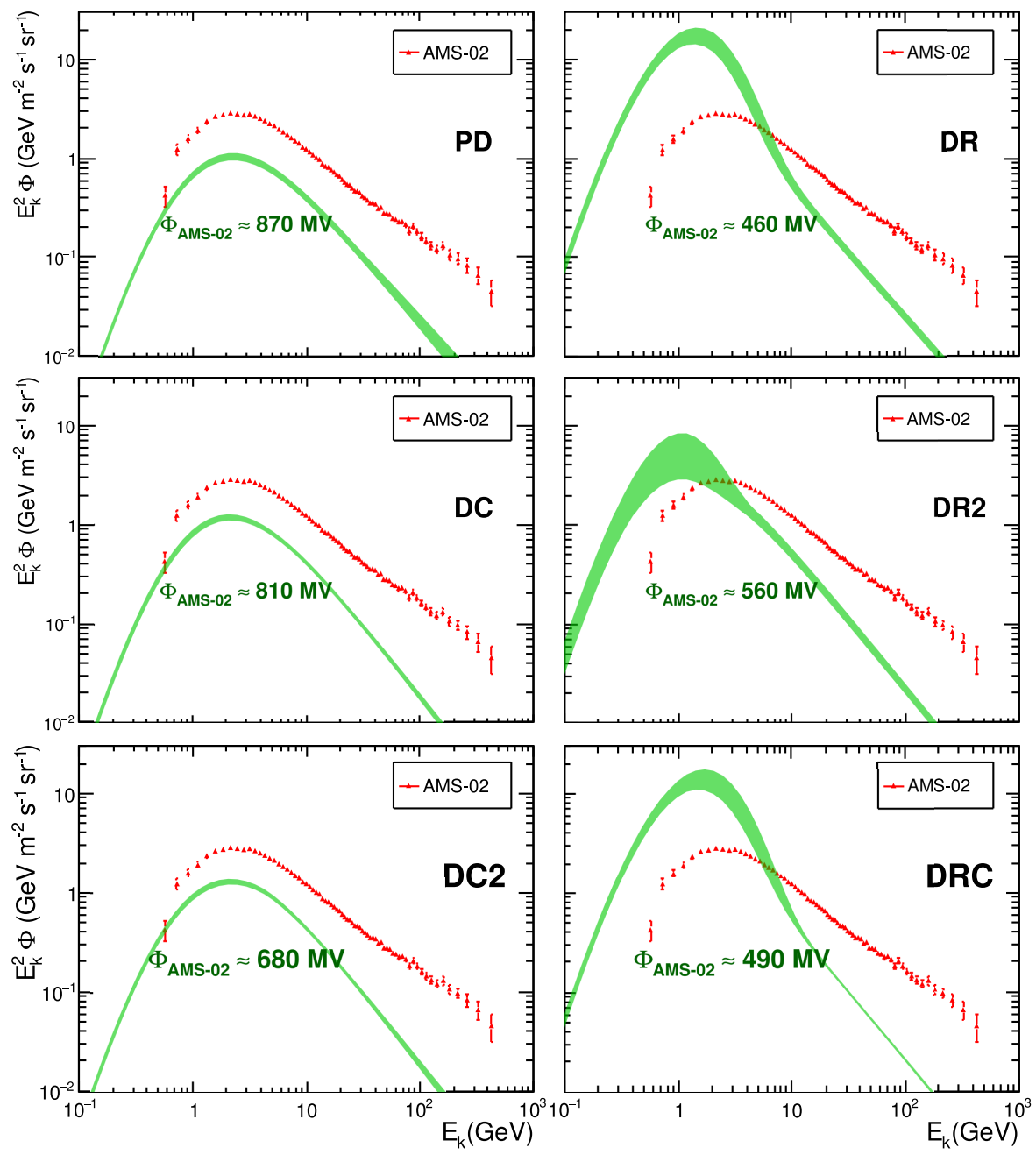

Figure 3: Predicted $2 \sigma$ bands of the positron spectra, compared with the AMS-02 measurements [22].

[10] M. Aguilar et al. [AMS Collaboration], Precision Measurement of the Boron to Carbon Flux Ratio in Cosmic Rays from 1.9 GV to 2.6 TV with the Alpha Magnetic Spectrometer on the International Space Station, Phys. Rev. Lett. 117 (2016) 231102.

[11] O. Adriani et al., Time dependence of the proton flux measured by PAMELA during the July 2006 December 2009 solar minimum, Astrophys. J. 765 (2013) 91 [arXiv:1301.4108].

[12] M. Aguilar et al. [AMS Collaboration], Precision Measurement of the Proton Flux in Primary Cosmic Rays from Rigidity 1 GV to 1.8 TV with the Alpha Magnetic Spectrometer on the International Space Station, Phys. Rev. Lett. 114 (2015) 171103.

[13] Q. Yuan, S. J. Lin, K. Fang and X. J. Bi, Propagation of cosmic rays in the AMS-02 era, Phys. Rev. D 95 (2017) 083007 [arXiv:1701.06149].

[14] S. Sharma, Markov Chain Monte Carlo Methods for Bayesian Data Analysis in Astronomy, arXiv:1706.01629

[15] A. Lewis and S. Bridle, Cosmological parameters from CMB and other data: A Monte Carlo approach, Phys. Rev. D 66 (2002) 103511 [astro-ph/0205436]. 


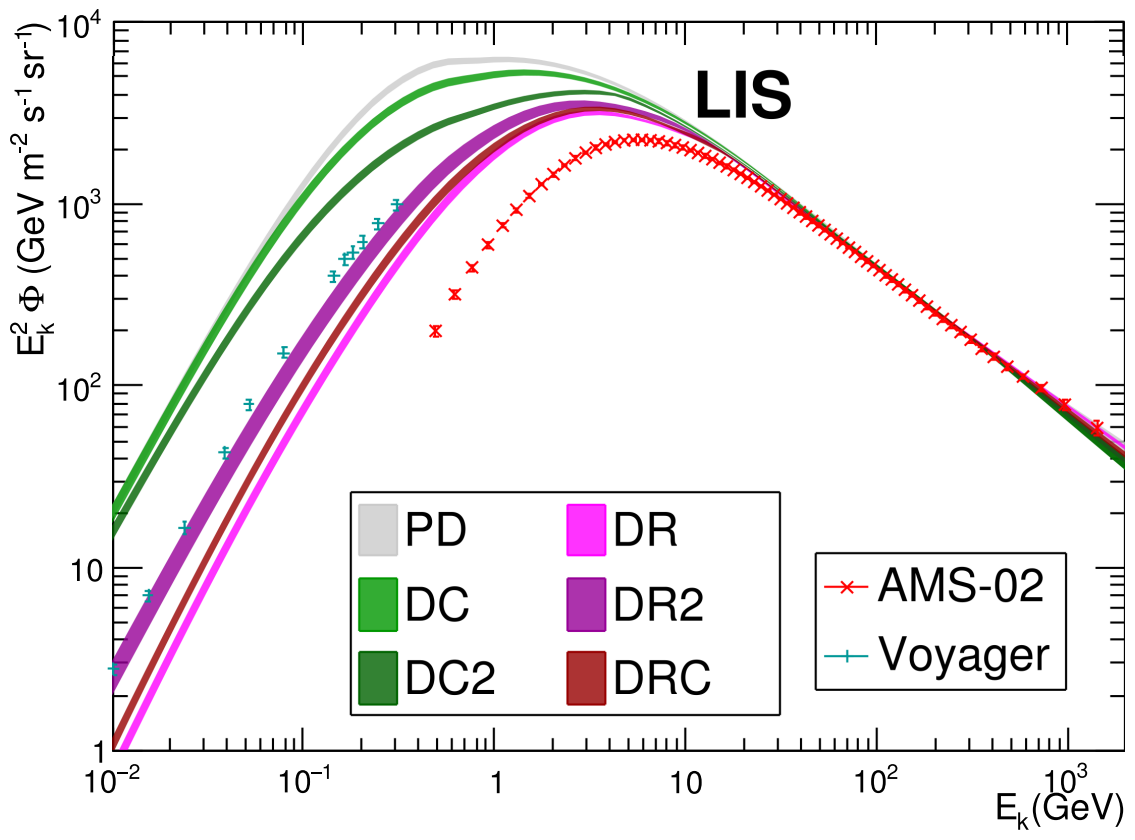

Figure 4: The LIS proton spectra from all the models in this work compared with the data of Voyager 1 [23] and AMS-02 [12].

[16] A. W. Strong and I. V. Moskalenko, Propagation of cosmic-ray nucleons in the galaxy, Astrophys. J. 509 (1998) 212 [astro-ph/9807150].

[17] I. V. Moskalenko and A. W. Strong, Production and propagation of cosmic ray positrons and electrons, Astrophys. J. 493 (1998) 694 [astro-ph/9710124].

[18] J. Liu, Q. Yuan, X. Bi, H. Li and X. Zhang, A Markov Chain Monte Carlo Study on Dark Matter Property Related to the Cosmic $e^{ \pm}$Excesses, Phys. Rev. D 81 (2010) 023516 [arXiv:0906.3858].

[19] J. Liu, Q. Yuan, X. J. Bi, H. Li and X. Zhang, CosRayMC: a global fitting method in studying the properties of the new sources of cosmic $e^{ \pm}$excesses, Phys. Rev. D 85 (2012) 043507 [arXiv:1106.3882].

[20] V. S. Ptuskin, I. V. Moskalenko, F. C. Jones, A. W. Strong and V. N. Zirakashvili, Dissipation of magnetohydrodynamic waves on energetic particles: impact on interstellar turbulence and cosmic ray transport, Astrophys. J. 642 (2006) 902 [astro-ph/0510335].

[21] L. J. Gleeson and W. I. Axford, Solar Modulation of Galactic Cosmic Rays, Astrophys. J. 154 (1968) 1011.

[22] M. Aguilar et al. [AMS Collaboration], Electron and Positron Fluxes in Primary Cosmic Rays Measured with the Alpha Magnetic Spectrometer on the International Space Station, Phys. Rev. Lett. 113 (2014) 121102.

[23] A. Cummings et al., Galactic Cosmic Rays in the Local Interstellar Medium: Voyager 1 Observations and Model Results, Astrophys. J. 831 (2016) 18.

[24] J. S. Niu and T. Li, Galactic cosmic-ray model in the light of AMS-02 nuclei data, arXiv:1705.11089 